\title{
La formation des oblats, missionnaires dans le Nord-Ouest canadien
}

\section{Claude Champagne}

Volume 56, 1989

Changements culturels et éducation de la foi

Cultural Change and Education of the Faith

URI : https://id.erudit.org/iderudit/1006953ar

DOI : https://doi.org/10.7202/1006953ar

Aller au sommaire du numéro

Éditeur(s)

Les Éditions Historia Ecclesiæ Catholicæ Canadensis Inc.

ISSN

0318-6172 (imprimé)

1927-7067 (numérique)

Découvrir la revue

Citer cet article

Champagne, C. (1989). La formation des oblats, missionnaires dans le

Nord-Ouest canadien. Sessions d'étude - Société canadienne d'histoire de l'Église catholique, 56, 21-33. https://doi.org/10.7202/1006953ar

Tous droits réservés @ Les Éditions Historia Ecclesiæ Catholicæ Canadensis Inc., 1989
Ce document est protégé par la loi sur le droit d'auteur. L'utilisation des services d’Érudit (y compris la reproduction) est assujettie à sa politique d'utilisation que vous pouvez consulter en ligne.

https://apropos.erudit.org/fr/usagers/politique-dutilisation/ 


\title{
La formation des oblats, missionnaires dans le Nord-Ouest canadien
}

\author{
Claude Champagne, o.m.i. \\ Institut des sciences de la mission \\ Université Saint-Paul \\ Ottawa
}

Les Oblats, engagés dans la mission auprès des Amérindiens et des Métis du Nord-Ouest canadien, ont cherché à transmettre la foi catholique, ce qu'ils ont appelé évangélisation, et à transformer la vie à la lumière de cette foi, ce qu'ils ont désigné sous le nom de christianisation. Quels ont été les modèles acquis lors de leur formation initiale? Quel a été le contenu missionnaire de l'enseignement dispensé dans les maisons de formation? Y a-t-il eu une formation spécifique pour les candidats à la mission auprès de groupes culturels différents de leur propre communauté? ${ }^{1}$ Ce sont là les questions auxquelles nous chercherons à répondre par cette communication.

Les Oblats de Marie-Immaculée, fondés par Eugène de Mazenod, en 1816 , en vue des missions populaires pour rechristianiser la campagne française, acceptent l'invitation de l'évêque de Montréal, Mgr Ignace Bourget, et arrivent au Canada, la même année, en 1841. Jusque-là, les Oblats n'avaient œuvré qu'en France. Ils mettent pied dans le Nord-Ouest, à Saint-Boniface, en $1845^{2}$. S'ils ne sont que deux en 1845 , à la fin de 1861 , le Nord-Ouest en compte 30, tandis que l'Orégon et la Colombie Britannique en ont $12^{3}$. En 1898, le Nord-Ouest est divisé en plusieurs

${ }^{1}$ Voir l'article de Jean-Claude BAUMONT, Jacques GADILLE et Xavier de MONTCLOS, L'exportation des modèles de christianisme français à l'époque contemporaine. Pour une nouvelle problématique de l'histoire missionnaire, dans Revue d'Histoire de l'Église de France 63 (1977), p. 10.

2 Donat LEVASSEUR, Histoire des Missionnaires Oblats de Marie Immaculée: essai de synthèse, I (1815-1898), Montréal, Maison Provinciale, 1983, p. 121-123; p. 132-133.

3 En 1861 , le personnel oblat du Nord-Ouest comprend 2 évêques, 20 pères et 8 frères tandis que l'Orégon et la Colombie Britannique ont 8 pères et 4 frères. LEVASSEUR, $o p$. cit., p. $138,143$. 
vicariats qui totalisent 238 Oblats $^{4}$. Enfin, en 1947, les provinces oblates du Manitoba, de l'Alberta-Saskatchewan et de Saint Peter (Colombie Britannique) comptent 151 Oblats consacrés à la mission auprès des Amérindiens, tandis que les vicariats apostoliques (Grouard, Mackenzie, Whitehorse, Prince Rupert, Keewatin et Baie d'Hudson) ont 354 Oblats'. Si les responsabilités des Oblats se modifient au $\mathrm{XX}^{\mathrm{e}}$ siècle, ils accordent encore une priorité aux missions indiennes, mais il demeure plus difficile de déterminer le nombre d'Oblats vraiment engagés dans ce ministère auprès des Amérindiens.

Les deux premiers Oblats dans le Nord-Ouest étaient de nationalités différentes: Pierre Aubert était français tandis qu'Alexandre Taché était d'origine canadienne. Les missionnaires oblats auprès des Amérindiens ont été majoritairement d'origine française, comme le soulignent les statistiques données par $M^{\mathrm{gr}}$ Vital Grandin pour le diocèse de Saint-Albert, en 1890: sur 31 Oblats, 20 sont Français, 7 Canadiens-français, 2 Belges, un Allemand et un Métis 6 . Selon les chiffres établis par Donat Levasseur, en 1899 , les Oblats d'origine française comptent pour $54,9 \%$ tandis que ceux du Canada sont 15,7\%. Sur les 479 Oblats à l'œuvre dans les vicariats de mission, 321 sont originaires de France et 63 du Canada. Les autres groupes sont beaucoup moins importants? .

Quels furent les principaux lieux de la formation initiale de ces Oblats, missionnaires dans le Nord-Ouest canadien? Le premier scolasticat fut établi à Marseille en 1827; en 1854, il est situé à Montolivet, en banlieue de Marseille. Il est transporté à Autun en 1862. Les expulsions de 1880 vont conduire le scolasticat en Irlande, en Hollande et enfin en Belgique, où il s'établissait d'une façon définitive à Liège en 1891. Au Canada, les Oblats ouvrent le scolasticat d'Ottawa en 1848. Viendront par la suite deux autres maisons de formation: le scolasticat de Rome, établi définitivement en 1887 et celui de Hünfeld, en Allemagne, inauguré en 18978. D'autres maisons de formation s'ouvriront pendant le $\mathrm{XX}^{\mathrm{e}}$ siècle.

${ }^{4}$ En 1898 , Saint-Boniface a un archevêque, 32 pères et 14 frères pour un total de 47 Oblats. Le vicariat d'Athabaska-Mackenzie, 62 Oblats dont 2 évêques, 30 pères et 30 frères; la Saskatchewan, 29 Oblats dont un évêque, 18 pères et 10 frères; Saint-Albert, 57 Oblats, soit 2 évêques, 28 pères et 27 frères; enfin, en Colombie Britannique, 43 Oblats, soit 2 évêques, 25 pères, 2 scolastiques et 14 frères. LEVASSEUR, op. cit., p. 232, 237, 246 et 251.

5 Donat LEVASSEUR, Histoire des Missionnaires Oblats de Marie Immaculée: essai de synthèse, II (1898-1985), Montréal, Maison Provinciale, 1986, p. 116, 131, 137, 150, 158, $163,172,176$.

6 Claude CHAMPAGNE, Les débuts de la mission dans le Nord-Ouest canadien. Ottawa, Éd. de l'Université d'Ottawa, 1983, p. 236.

7 Donat LEVASSEUR, op. cit. I, p. 307-308.

8 Donat LEVASSEUR, op. cit. I, p. 180-181. 
Si peu d'études ont encore été faites sur les maisons de formation, on sait, qu'en général, dans la France du $\mathrm{XIX}^{\mathrm{e}}$ siècle, les programmes d'études des séminaires diocésains et des scolasticats missionnaires sont les mêmes: les manuels de théologie sont identiques 9 . Cette conclusion se voit confirmée pour la période qui va jusqu'à la mort du fondateur des Oblats, en 1861:

Le Fondateur tenait à ce que l'on ne néglige pas la formation ecclésiastique des futurs missionnaires. En général il préférait assigner l'obédience à la dernière minute; les étudiants restaient ainsi dans l'ignorance complète de leur destination et recevaient tous la même formation générale ${ }^{10}$.

Cette affirmation de Quéré est confirmée par Yvon Beaudoin qui a étudié la première maison de formation oblate, le scolasticat de Marseille, qui fait également office de grand séminaire du diocèse de Marseille:

On ne choisissait pour les missions que ceux qui les désiraient et dont les vertus et l'esprit de sacrifice donnaient de sérieuses garanties de persévérance et de courage. En général, ils ne connaissaient leur obédience qu'au dernier moment, mais tous, comme l'affirme Louis Soullier, étaient «comme l'oiseau sur la branche», prêts à s'envoler au premier signal. S'ils ne recevaient, comme les autres Oblats, qu'une éducation de base qui comprenait en outre l'étude de l'anglais, obligatoire pour tous, ils s'exerçaient du moins à l'apostolat de la prière comme l'attestent leurs écrits et l'existence d'une association de piété dans ce but ${ }^{11}$.

Quéré et Beaudoin concluent qu'il n'y a pas de formation particulière pour les missionnaires destinés à d'autres groupes ethniques. Ils ne recevaient leur obédience qu'à la fin de leur programme d'études. Les formateurs ont surtout insisté sur le désir des missions étrangères et sur les vertus nécessaires à ce genre de vie.

Pour la période qui suit la mort du fondateur des Oblats, un périodique intitulé Missions des Oblats de Marie Immaculée, publié presque sans interruption de 1862 à 1972 , est un témoin de choix de la vie de la congrégation. Or, l'Index, établi récemment, ne fait pas allusion à une formation particulière pour les missionnaires oblats à l'étranger avant les années 1930. C'est en effet en 1931 qu'est annoncée la création d'une chaire de missiologie à Ottawa et que le P. Albert Perbal, missiologue oblat, publie un premier article sur la science des missions ${ }^{12}$. C'est en

9 Xavier de MONTCLOS, La vie spirituelle en France au XIXe siècle et l'élan missionnaire, dans Les réveils missionnaires en France, du Moyen Âge à nos jours, $\left(\mathrm{XII}^{\mathrm{c}}-\mathrm{XX}^{\mathrm{c}}\right.$ siècles), Paris, Beauchesne, (1984), 322-326.

10 Martin QUÉRÉ, M ${ }^{g r}$ de Mazenod et les missions étrangères, manuscrit, Rome, 1958, p. 194.

11 Yvon BEAUDOIN, Le grand séminaire de Marseille (et scolasticat oblat) sous la direction des Oblats de Marie Immaculée 1827-1862, Ottawa, Éd. des Études Oblates, 1966, p. 143. (Archives d'histoire oblate 21).

12 Missions des o.m.i. 65 (1931), p. 487, 640-656. 
1947 que le père Joseph-Étienne Champagne, missiologue d'Ottawa, va publier son Manuel d'Action Missionnaire ${ }^{13}$.

Peu d'études ont encore été réalisées sur le programme d'enseignement des différentes maisons de formation oblate. Seul, le scolasticat de Marseille a jusqu'ici été l'objet d'une thèse de doctorat du P. Beaudoin, déjà citée plus haut. On peut présumer que l'enseignement donné à cet endroit s'est maintenu à Montolivet, à Autun et aux autres endroits où le scolasticat a été établi. De plus, quelques-unes des grandes figures qui ont illustré l'histoire missionnaire du Nord-Ouest ont reçu leur formation initiale à Marseille. Notons en particulier le fondateur de la mission de la Rivière-Rouge, en 1845, Pierre Aubert, de même que quatre évêques qui ont œuvré dans ce territoire: Henri Faraud, Louis D'Herbomez, Vital Grandin et Paul Durieu, sans oublier sept missionnaires qui ont travaillé dans le Nord-Ouest jusqu'au début du $\mathrm{XX}^{\mathrm{e}}$ siècle $^{14}$. Nous analyserons donc cette formation initiale donnée aux candidats à la mission dans le Nord-Ouest.

À Marseille, les professeurs avaient reçu une formation limitéc; ils n'avaient pas eu le temps de se spécialiser; trop facilement n'importe qui enseignait n'importe quoi. Ils n'étaient donc pas préparés à affronter un monde en changement et une culture différente de celle de l'Ancien Régime en France. Ils n'étaient pas en mesure d'apporter des réponses évangéliques aux nouvelles questions posées par l'évolution rapide de la société et par le contact avec d'autres cultures et d'autres groupes ethniques, comme les Amérindiens du Nord-Ouest canadien.

Chez les Oblats, l'obéissance au pape faisait l'objet d'un article de la Règle; en pratique, les professeurs du scolasticat furent ultramontains et choisirent les manuels de théologie en conséquence.

Si la durée des études est généralement de quatre ans, plusieurs Oblats destinés aux missions étrangères ne sont restés au scolasticat qu'un ou deux ans, soit que leurs études fussent déjà commencées ailleurs ou qu'ils aient été envoyés en Angleterre ou au Canada et, parfois même, sur le lieu de leur mission pour terminer leur préparation au ministère. Le

\footnotetext{
13 Publié à Ottawa, aux Éditions de l'Université, 843p.

14 Les quatre évêques ont œuvré de nombreuses années dans le Nord-Ouest: Henri Faraud (1847-1890), Louis D'Herbomez (1850-1890), Vital Grandin (1854-1902) et Paul Durieu (1854-1899). Les sept autres missionnaires sont Alexis André (1861-1893), FrançoisXavier Bermond (1846-1856), Léon Fouquet (1859-1912), Charles Pandosy (1847-1891), René Rémas (1852-1901), Pierre Richard (1854-1907) et Valentin Végreville (1852-1903). On pourra consulter le Dictionnaire biographique des Oblats de Marie-Immaculée au Cana$d a$ de Gaston CARRIÈRE, 3 vol., Ottawa, Éd. de l'Université d'Ottawa, 1976-1979. Les années entre parenthèses indiquent le temps de présence dans l'Ouest canadien. Yvon BEAUDOIN, op. cit. 171-178.
} 
fondateur, $\mathrm{M}^{\mathrm{gr}}$ de Mazenod, insistait pour qu'on assure aux jeunes Oblats la science indispensable au ministère, avant l'ordination. Si plusieurs scolastiques, destinés aux missions lointaines, ont fait des études hâtives, ils auraient tout de même vu les principaux traités. Ces envois en mission, à la hâte, conduisirent certains Oblats à conclure que l'instruction n'était pas tellement nécessaire pour aller aux missions étrangères ${ }^{15}$.

Quels sont les ouvrages théologiques utilisés à Marseille? Les principaux traités dogmatiques, l'Incarnation et la Trinité, sont vus dans la Somme théologique de saint Thomas d'Aquin ${ }^{16}$. Des exemplaires des Institutiones theologica ad usum seminariorum en six volumes de JeanBaptiste Bouvier ont été achetés pour Marseille ${ }^{17}$. Cet ouvrage vit sa première édition complète en 1834 et en connut quinze autres. Certains passages, de tendance gallicane, sont corrigés à partir de la huitième édition, en 1853. Les directeurs du séminaire de Marseille, ultramontains, ne purent être complètement satisfaits du texte de Bouvier. Ils publièrent ainsi deux manuels anonymes, mais attribués au Père Joseph Fabre, sur la vraie religion révélée: Tractatus de Religione revelata maxime christiana, en 1852, et sur la vraie Église du Christ: Tractatus de Vera Christi Ecclesia, en $1853^{18}$.

Nous chercherons maintenant à déterminer le modèle d'Église, la représentation des protestants et des non-chrétiens et, enfin, la conception de la mission, véhiculés dans les manuels utilisés au scolasticat de Marseille. Nous retenons les deux publications anonymes publiées à Marseille sur la vraie religion et l'Église, ainsi que les Institutiones theologica de Bouvier. L'analyse de ce dernier ouvrage se fait à partir de la $4^{\mathrm{e}}$ édition, celle de 1841. Comparaison faite avec l'édition de 1861, aucun des textes cités n'a été modifié.

Le traité de Vera Christi Ecclesia nous aide à préciser la vision ecclésiologique offerte aux premiers missionnaires oblats engagés dans le Nord-Ouest canadien. L'ouvrage se divise en trois grands chapitres: le premier porte sur l'institution de l'Église, ses notes et la recherche de la vraie Église de Jésus-Christ; le deuxième, sur le pouvoir dans l'Église; et le dernier, très bref, sur les membres de l'Église ${ }^{19}$.

Dès le début, l'auteur affirme que l'Église est instituée par le Christ: sa fin première est de conserver la vraie foi et de la mettre en pratique; la fin secondaire: le salut éternel qu'on ne peut obtenir hors de l'Église. Or,

15 Claude CHAMPAGNE, op. cit., p. 30-31.

16 Ibid., p. 36.

17 BEAUDOIN, op. cit., p. 95.

18 Claude CHAMPAGNE, op. cit., p. 17, 32 et 36.

19 Ibid., p. 36-37. 
pour ce faire, il faut observer intégralement la religion chrétienne telle que révélée par le Christ et propagée par les Apôtres. Rien ne manifeste encore un éveil au contexte historique et culturel de cette révélation de la religion chrétienne et de sa propagation. Or, comme plusieurs Églises se réclament du Christ, il faut chercher la véritable Église du Christ à partir des notes de la vraie Église: une, sainte, catholique et apostolique. Pour prouver que ces quatre notes s'appliquent exclusivement à l'Église catholique, l'auteur juge nécessaire de démontrer comment les communautés non catholiques ne jouissent pas de l'unité, de la sainteté, de la catholicité et de l'apostolicité. Cette manière d'aborder la question de l'Église va peser sur les relations entre catholiques et protestants ${ }^{20}$.

Le reste du manuel nous met en présence d'une Église fortement structurée où l'uniformité est perçue d'une manière très positive. L'Église présentée aux premiers missionnaires est sous le signe de l'autorité; on met l'accent sur les structures, mais la dimension spirituelle demeure dans la pénombre. La diversité culturelle n'y trouve pas sa légitimité. Rien n'y est dit de la dynamique missionnaire ${ }^{21}$.

Quant aux protestants, les manuels de Fabre et de Bouvier les qualifient d'hérétiques. S'il n'est pas exclu de rencontrer des protestants de bonne foi 22 , il n'en demeure pas moins que l'hérétique, rejetant quelquesunes des vérités révélées par Dieu, rejette l'autorité de Dieu ${ }^{23}$ affirme Fabre, tandis que Bouvier parle des vices opposés à la foi: l'hérésie est une foi acceptée, puis en partie abandonnée 24 .

Tout le traité de l'Église se fait de manière apologétique contre les protestants. Les théologiens veulent prouver que l'Église catholique romaine est la vraie et, par conséquent, que les autres sont fausses. L'usage des notes d'unité, de sainteté, de catholicité et d'apostolicité va conduire les ecclésiologues à tenter d'établir que ces notes s'appliquent à l'Église catholique et seulement à elle; cette démarche comporte la démonstration que les notes ne se retrouvent ni chez les protestants, ni chez les orthodoxes. Cette attitude défensive de la théologie catholique explique la réaction négative des missionnaires oblats face aux ministres protestants qu'ils vont côtoyer en Amérique du Nord. Ces Oblats sont difficilement en mesure de reconnaître le zèle et le dévouement des missionnaires des autres confessions.

\footnotetext{
20 Ibid., p. 37-38.

21 Ibid., p. 38-40.

22 (FABRE), Ecclesia, p. 10.

23 Ibid.

${ }^{24}$ BOUVIER, Institutiones theologic a, p. 5. 35.
} 
Ainsi, à l'objection voulant que les réformés aient leurs apôtres, leurs saints et leurs martyrs, Fabre répond:

Ils ont quelques apôtres seulement adhérant à leur credo, si ce n'est ceux que la liberté d'action incite au crime; leurs communautés possèdent des saints renommés ni pour l'éminence de leur vertu, ni pour l'éclat de leurs miracles mais d'une honnêteté irréprochable et cela en vertu de ce qui leur reste de la religion catholique ou d'un bon naturel et en petit nombre seulement; ils ont des martyrs, mais tout à fait différents des nôtres, d'une part quant à leur nombre, et d'autre part quant à leur patience, leur amour des ennemis et quant à leurs autres vertus 25 .

Pour démontrer la sainteté de l'Église catholique romaine, Bouvier apporte comme preuve les miracles, le zèle pour la propagation de la foi et la conversion de ceux qui sont dans l'erreur; comme les protestants sont eux aussi engagés dans l'activité missionnaire, Bouvier fait le commentaire suivant:

Les protestants contemporains ont voulu imiter les catholiques; ils ont aussi leurs missions, et ils dépensent d'énormes sommes d'argent pour répandre parmi toutes les nations l'Écriture sainte traduite en langues vulgaires; mais leurs missionnaires n'ont pas encore fait montre du zèle, de la piété, de l'abnégation de soi et des autres vertus hérö̈ques qui brillent chez les missionnaires catholiques; ils n'ont jamais non plus accompli de miracles et n'ont pas engendré de chrétiens irréprochables du point de vue de la doctrine, stables dans la foi et parfaits dans le comportement. De plus Dieu ne peut pas permettre qu'une société non sainte, schismatique ou hérétique puisse remplir seule sans interruption la tâche de prêcher l'Évangile26.

Dieu ne peut permettre que de telles sociétés puissent mener à bien la tâche de prêcher l'Évangile: les missions protestantes ne peuvent comporter d'éléments positifs.

Bouvier considère enfin les conversions de protestants à l'Église catholique et le passage de catholiques aux autres «sectes» de la manière suivante:

25 (FABRE), Ecclesia, p. 49-50.

26 BOUVIER, op. cit., p. 1, 310. Si, en 1947, le P. Joseph Champagne suit l'évolution de l'ecclésiologie catholique en parlant de l'Église comme corps du Christ, la vision des protestants ne s'est pas encore beaucoup modifiée depuis le siècle précédent. «Une Église dissidente est une société organisée dont le culte, les dogmes et les lois sont puisés dans la Révélation chrétienne, mais qui a refusé de croire en un ou plusieurs dogmes enseignés par l'Église du Christ (les protestants du XVI ${ }^{e}$ siècle, [...]. Le terme "Églises chrétiennes» appliqué aux Églises dissidentes peut prêter à équivoque. Il n'y a en effet qu'une seule Église qui soit vraiment chrétienne, c'est la seule et unique Église du Christ, l'Église catholique. Les Eglises dissidentes ne sont pas «le corps du Christ» et elles n'ont en tant qu'Églises hérétiques ou schismatiques, ni mission, ni pouvoir pour appliquer aux hommes les fruits dc la Rédemption. L'emploi du mot «chrétienne» à leur endroit indique l'origine évangélique de leur culte, de leurs lois et de leur foi surtout. Culte et foi qui se réclament du Christ, mais plus ou moins défigurés en réalité» (CHAMPAGNE, Manuel d'Action Missionnaire), Ottawa, Éd. de l'Université d'Ottawa, 1947, 843p. 
L'Église romaine est considérée comme tellement sainte et tellement divine que les quelques hommes qui soumettent la question à un examen plus sérieux, sont disposés à refréner leurs passions, désireux de vivre plus saintement et, exposés à un danger, recherchent avec plus de diligence le salut éternel, abandonnent chaque jour les autres sectes pour chercher refuge en elle; et au contraire, si nous voyons parfois quelques membres de l'Église romaine chercher refuge dans les autres Églises, c'est qu 'ils sont, du moins ordinairement, alléchés par les séductions du monde, esclaves de leurs passions et se proposent de vivre plus librement, tels les moines qui ne peuvent supporter la discipline, les prêtres qui veulent se marier... etc... Donc tous sont persuadés que l'Église romaine l'emporte en sainteté sur les autres sociétés chrétiennes ${ }^{27}$.

Reste à préciser quels types de relations peuvent être entretenues avec «les hérétiques et les schismatiques». S'il n'y a pas de difficultés en ce qui a trait au domaine de la cité, il ne peut y avoir de communication active dans le domaine des choses sacrées: participation à des cérémonies, etc. 28 .

Bouvier touche enfin De disputatione cum hariticis. Même un «docte laic» est invité à s'engager en vue du salut du prochain et de l'honneur de la foi. Les clercs peuvent eux aussi défendre l'honneur de la vérité, mais, dans tous les cas, il ne s'agit que de disputes et de polémiques ${ }^{29}$.

La vision plutôt négative des protestants, véhiculée dans les manuels de théologie du scolasticat de Marseille, ne préparait pas à l'ouverture et à la sympathie face à leur activité missionnaire dans le Nord-Ouest de l'Amérique du Nord.

Pour Bouvier, ce qui caractérise les non-chrétiens, c'est l'absence ou la carence de la foi ${ }^{30}$ : voilà pourquoi on désigne les non-chrétiens sous le nom d'infidèles. Parmi les types d'infidélités, l'auteur distingue celle dite négative, de celle où l'Évangile n'a jamais été prêché31. Ce type d'infidélité n'est pas coupable. Voici la situation de ces infidèles:

En conséquence, ceux qui trempent dans une infidélité de ce genre, ou ont commis quelque péché actuel, ou n'en ont pas commis; dans le premier cas, ils seront damnés, non pas à cause de leur infidélité qui n'est pas coupable, mais à cause de leur péché dont ils ne peuvent obtenir le pardon sans la foi; dans le second cas, ils sont dans le même état que les enfants non baptisés. Du reste il faut faire remarquer qu'il appert, en vertu de ce qui a été démontré dans le traité de la grâce, qu'une grâce, au moins virtuellement suffisante est donnée à tous les infidèles, grâce par le bon usage de laquelle ils peuvent obtenir la foi et la conversion à Dieu s'ils le veulent: ils ne périront donc pas sinon par leur faute ${ }^{32}$.

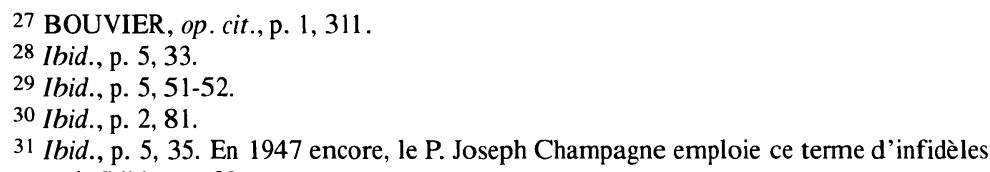
et de pays infidèles, p. 23.

32 Ibid., p. 5, 35. 
Parmi les infidèles, on compte les Juifs, les «Mahométans» et les païens qui englobent les idolâtres, les athées et les déistes qui ne furent pas baptisés ${ }^{33}$.

Dans le De religione revelata, Fabre veut montrer la nécessité de la révélation et établir que la vraie religion révélée est la chrétienne. Ce traité est dirigé à la fois contre ceux qui nient la possibilité de la révélation et contre les religions non chrétiennes tout comme le traité sur l'Église est tourné contre les chrétiens non catholiques.

Lorsqu'il s'agit d'affirmer l'existence de la révélation, l'auteur annonce qui sont les adversaires:

Nous luttons ici à la fois contre les mahométans, les païens et les juifs modernes dont les sectes, comme nous le montrerons, ne viennent pas de Dieu et contre les naturalistes, les déistes dont l'intention principale a toujours été de nier l'existence de la révélation ${ }^{34}$.

Pour montrer la nécessité de la révélation, l'auteur fait appel à l'expérience religieuse de tous les temps: polythéisme, cérémonies barbares, rites ridicules, absurdes et criminels, vols, homicides, incestes, adultères, prostitution, etc. ${ }^{35}$.

Fabre postule une révélation primitive et en voit la confirmation dans la croyance que l'on retrouve chez les peuples:

Auprès de peuples variés, nous trouvons non seulement la conviction universelle que Dieu est venu jadis parmi les hommes, que la religion a été apportée du ciel, et aussi les dogmes et les rites, dont la raison seule ne pouvait découvrir la vérité, dont l'uniformité indique en outre la même origine divine. Par conséquent, la connaissance de ceux-ci doit être attribuée de manière directe ou indirecte à une révélation primitive ${ }^{36}$.

On explique par révélation ce que la raison ne pouvait découvrir par ses propres forces. Combattant le rationalisme de l'époque, la théologie insistait sur l'impuissance de la raison et se trouvait en lutte contre l'homme sans foi ni grâce, c'est-à-dire contre le non-chrétien. La pensée traditionaliste affirmait qu'il ne pouvait y avoir de connaissance d'ordre religieux sans révélation et ainsi était amenée à affirmer une révélation primitive pour comprendre le monde religieux non chrétien. Mais cette religion primitive s'est corrompue:

33 Ibid., p. 5, 35-36.

34 (FABRE), Religinne, p. 83.

35 Ibid, p. 15-16.

36 Ibid., 92. Pour Vital Grandin, la religion traditionnelle des Dené a conservé des traces de la religion biblique, en particulier les récits de la Genèse: récits des origines, du déluge, mais aussi allusion aux géants. Claude CHAMPAGNE, op. cit., p. 82-83. 
La religion primitive devenue graduellement plus pure et plus parfaite, victime des interventions de la raison elle aussi en progrès, fut obscurcie et sombra dans le polythéisme ${ }^{37}$.

Après avoir affirmé l'existence de la révélation primitive, le caractère provisoire de la révélation judaïque et l'origine divine de la révélation chrétienne, Fabre consacre un article à démontrer que seule la religion chrétienne est vraie et qu'ainsi toutes les autres sont dans l'erreur. Parmi toutes les religions anciennes et modernes, un petit paragraphe consacré aux «B̄arbares» modernes semble viser les Iñdieñs d' Ammérique:

Les païens d'aujourd'hui qui n'admettent pas les erreurs mentionnées en admettent d'autres non moins grossières, qui varient selon la diversité des lieux. Ils sont attachés à l'idolâtrie, ils accomplissent souvent de très nombreux sacrifices humains, ils professent des principes moraux contraires à la raison et se montrent souvent à peine humains et sont enfoncés dans des erreurs presque si absurdes que les reproches adressés aux peuples anciens s'appliquent aussi à eux dans l'ensemble ${ }^{38}$.

Pour illustrer ce caractère inhumain, Fabre cite un exemple venant d'Amérique: «Les païens d'Amérique ensevelissaient avec le cadavre de la mère plusieurs foetus qu'ils tuaient avant la naissance à titre d'expiation ${ }^{39}$ ».

Les mœurs des païens frappent par leur absurdité et leur inhumanité. Et tout comme face aux protestants, il n'est pas possible de reconnaître des vertus chez les non-chrétiens:

La religion chrétienne a aussi enseigné et donné l'exemple du courage dans l'adversité. Dans les autres sectes, on peut repérer ici et là quelques exemples de courage, qui consistait en un vain désir d'une gloire immortelle ou en une préoccupation fanatique issue de l'obligation morale de mépriser jusqu'à un certain point le danger. Vraiment le courage des chrétiens joint à la prudence, à la tempérance et à la justice, c'est-à-dire escorté de toutes les vertus, non issu de la vanité, ni d'un transport fanatique mais du simple amour de Dieu, tolérait les dangers et les malheurs non seulement patiemment et sans crainte mais aussi avec joie ${ }^{40}$.

Les théologiens qui voulaient démontrer la vérité de la religion chrétienne ne pouvaient reconnaître une valeur positive chez ceux qui n'étaient pas chrétiens. Certes, tout n'est pas noir dans le tableau. Bouvier affirme: «L'homme sans la foi chrétienne peut accomplir une bonne action morale, et en conséquence tous les actes des infidèles ne sont pas nécessairement des péchés ${ }^{41}$.

\footnotetext{
${ }^{37}$ Ibid., p. 95.

38 Ibid., p. 213.

39 Ibid., p. 242.

40 Ibid., p. 244-245.

${ }^{41}$ BOUVIER, op. cit., p. 2, 291.
} 
Le non-chrétien peut faire le bien et à tous les infidèles est offerte la grâce indirectement suffisante ${ }^{42}$. L'auteur insiste sur la volonté de salut de Dieu: «Dieu veut sincèrement le salut de tous les hommes et il a préparé pour eux les moyens suffisants ${ }^{43}$ ». Le Christ est mort pour le salut de tous: «Le Christ est mort pour tous les hommes, dans l'intention de mériter pour chacun des grâces relativement suffisantes ${ }^{44}{ }^{»}$. L'intention universelle du salut est donc affirmée.

Mais Bouvier exprime également la nécessité de la foi et du baptême pour le salut; il distingue une nécessité de moyen et une nécessité de précepte. Est de nécessité de moyen ce qui ne peut être omis même sans culpabilité: sans le baptême, on ne peut obtenir le salut:

Le baptême est nécessaire à tous pour le salut d'une nécessité de moyen, en

fait pour les enfants en dehors du martyre, et en fait ou en désir pour les adultes ${ }^{45}$.

Bouvier parle aussi de nécessité de précepte pour des réalités qu'on ne peut omettre volontairement sans pécher, mais dont l'omission non coupable ne nuit pas au salut, comme la réception de l'Eucharistie ${ }^{46}$.

Reste à dire un mot sur les relations avec les infidèles et sur la tolérance des princes chrétiens face aux rites païens: «Pourvu qu'il n'y ait pas danger de perversion ou de scandale, il est permis de participer avec les païens à toutes les liturgies civiles ${ }^{47}$ ». Quant à la liberté de culte, les princes chrétiens doivent choisir le moindre mal:

Les liturgies des infidèles ne peuvent jamais être approuvées ou favorisées par les princes chrétiens mais il leur est parfois permis de les tolérer, si, par exemple, leur interdiction devait entraîner de plus grands maux ou empêcher de plus grands biens; de deux maux qui ne peuvent être évités, on doit bien sûr éviter le plus grand 48 .

Les non-chrétiens ne jouissent pas de la sympathie des théologiens. Certes, l'apologétique conduit à noircir ceux contre qui on s'affirme, particulièrement dans un traité comme le De vera Religione. Par ailleurs, on reconnaît que les non-chrétiens sont appelés au salut mérité par la mort du Christ; mais nombreux sont ceux qui n'ont jamais entendu la proclamation de l'Évangile. Il y a place pour la mission ou la conversion des infidèles.

On parle très peu de l'activité missionnaire dans les manuels théologiques des premiers missionnaires oblats dans l'Ouest canadien. $\mathrm{Si}$

\footnotetext{
42 Cf. Ibid., p. 2, 320.

43 Ibid., p. 2, 329.

44 Ibid., p. 2. 337.

45 Ibid., p. 2, 448.

46 Ibid., p. 5, 10.

47 Ibid., p. 5, 37.

48 Ibid., p. 5, 38.
} 
on a utilisé le zèle à propager la foi pour démontrer l'origine divine de l'Église catholique, quelques pages seulement sont consacrées aux missions dans le traité De Fide, lorsqu'on traite des vices contre la foi et de l'infidélité. On y relie la conversion des infidèles au précepte du Christ.

Bien que l'infidélité négative ne soit pas en soi un péché mais une peine due au péché, à savoir celui d'Adam, on constate néanmoins que le précepte de prêcher l'Évangile à toute créature a été donné par le Christ aux Apôtres et à leurs successeurs (Marc XVI, 15); c'est pourquoi nous devons être embrasés du zèle ardent pour amener les infidèles de toute origine à la foi chrétienne, en leur démontrant qu'autrement ils cheminent hors de la voie du salut. De là cette louable coutume, permanente dans l'Église romaine d'envoyer des missionnaires aux infidèles de toutes les religions du monde entier 49 .

L'activité missionnaire du $\mathrm{XIX}^{\mathrm{e}}$ siècle se relie à ce mouvement intime fondé sur le commandement évangélique et repris à travers les siècles. Les théologiens ne manifestent pas une conscience des formes diverses qu'a pris cette activité selon les différents contextes socio-culturels.

Bouvier précise que seule la persuasion peut conduire à la conversion; la contrainte n'a pas sa place dans la mission. Il en explique la raison:

La nature de la foi, qui est un acte de l'esprit, et vraiment pas du corps, suppose une vraie liberté, et le Christ quand il dit: «Qui croira, sera sauvé: qui ne croira pas d'autre part sera condamné», suppose que chacun a la faculté de choisir 50 .

Mais Bouvier croit que les princes chrétiens peuvent légitimement interdire les cultes idolâtres si ces derniers offensent le vrai Dieu et scandalisent le peuple ${ }^{51}$. De plus, l'intervention des puissances occidentales dites chrétiennes dans les pays non chrétiens se justifie pour la protection des missionnaires et par le droit de l'Église de prêcher l'Évangile dans le monde entier 52 . Mais le missionnaire ne peut forcer les infidèles à accepter sa prédication:

Mais il sera entièrement illicite et étranger à l'esprit évangélique que les missionnaires s'avancent avec des armes ou des soldats, afin de contraindre les infidèles à écouter la prédication de la doctrine de la foi; en effet, les armes des hommes apostoliques doivent être la patience, la paix, le jeûne, les veilles, la chasteté, la charité, la vérité, la douceur, comme l'enseigne le B. Paul, II Cor, vi, 4 et suiv. d'où les paroles du Christ, Luc XIV, 23, force-les à entrer doivent être prises dans un sens moral et comprises comme faisant allusion à la contrainte par les moyens de persuasion ${ }^{53}$.

49 Ibid., p. 5, 36.

50 Ibid., p. 5, 36.

51 Ihid., p. 5, 36-37. $\mathrm{M}^{\mathrm{gr}}$ Grandin formulera le souhait que le gouvernement canadien travaille à faire cesser la polygamie et certaines «superstitions cruelles en usage chez certaines tribus indiennes». Cf. Claude CHAMPAGNE, op. cit., p. 48, note 28.

52 Cf. BOUVIER, op. cit., p. 5, 37.

53 Ibid., p. 5, 37. 
C'est là le seul passage des Institutiones theologica de Bouvier qui porte explicitement sur l'activité missionnaire. Si l'activité missionnaire était très importante dans la France catholique du XIX $\mathrm{X}^{\mathrm{e}}$ siècle, la réflexion sur cette activité tardait à venir.

Le Grand Séminaire et scolasticat de Marseille formaient à la fois des missionnaires pour les campagnes de France et pour les missions lointaines. On y offrait l'éducation de base: les missionnaires du Nord-Ouest canadien n'y reçurent pas de formation particulière en vue d'une activité dans un groupe de cultures et de religions différentes. Les manuels de théologie, en usage à l'époque, offraient un modèle d'Église fortement structurée, en lutte contre les protestants et les infidèles. Si ces manuels faisaient allusion à l'activité missionnaire et à la conversion des infidèles, la réflexion systématique sur la mission n'avait pas encore vu le jour. Elle n'apparut qu'au début du XX $\mathrm{X}^{\mathrm{e}}$ siècle et ce n'est qu'au début des années '30 que le périodique Missions des O.M.I. fait écho à la nouvelle science missiologique. 